\title{
LIFESTYLE AND DIETARY FACTORS ASSOCIATING WITH DEMENTIA STATUS IN THE COMMUNITY-DWELLING ELDERLY AGED 65 AND OLDER IN A SUBURBAN TOWN OF TOKYO
}

\author{
CHISAKO YAMAMOTO, TANJI HOSHI
}

\begin{abstract}
:
Background: Dementia is a priority health issue worldwide. Building dementia-friendly communities was discussed at the 32nd International Conference of Alzheimer's Disease International in April 2017 and followed in May by the adoption of Global Action Plan on the Public Health Response to Dementia by the 70th World Health Assembly (WHO). An unhealthy lifestyle and diet are likely to cause many diseases. Objectives: This study aims to clarify lifestyle and dietary factors associating with dementia status in the community-dwelling elderly aged 65 and older in a suburban town of Tokyo and to clarify gender differences in health behavior. Methods: Self-administered questionnaires were mailed to 2,069 elderly people in February 2004 and 1,538 responses were returned by addressees or proxies (response rate; $74.3 \%$ ). Institutionalized ones were excluded. Analysis subjects were comprised of 52 people with dementia (PWD), 173 people with probable dementia (PPD) and 1,211 cognitively intact people (CIP). Mean age (sd) was 74.03 (6.55) in men and 75.56 (7.27) in women. Descriptive statistics, a chi-square, Kruskal-Wallis and Mann-Whitney U tests and Bonferroni's multiple comparisons were performed in men and women, respectively. Significance was set at 0.05 (0.017 after Bonferroni correction). Lifestyle items included smoking, alcohol drinking, cooking, breakfast eating, walking/exercise, sleep duration per day including nap, going out, daytime lying duration in bed and the number of hobbies. Dietary items included meat/poultry, soybean products, eggs, oily fish, dairy products, fruits, vegetables, salted fish, deep-/stir-fried food, miso soup/soup, pickled ume plums and pickled vegetables. Results: Significant differences were detected in most lifestyle items except smoking and breakfast eating in men and women. As for dietary items, a significant difference was found only in pickled vegetables in men, however, in women significant differences were detected in items except eggs, salted fish, heavily seasoned food and pickled ume plums. Conclusion: Significant differences in lifestyle were detected in the same items in the CIP men and women. Exercise, social and mental activities like going out and hobbies, moderate alcohol drinking and dietary items above are recommended to prevent or delay the onset of dementia by Alzheimer's Society. The results of this study showed the same implications. As for dietary items, significant differences were observed in nine out of 13 items in women, while only one item was clarified in men. Health behavior seems to be better in women.
\end{abstract}

\section{Keywords:}

The community-dwelling elderly aged 65 and older, lifestyle and dietary habits, dementia status, risk reduction, health behavior, survey data.

JEL Classification: $110,118,119$ 


\section{Authors:}

CHISAKO YAMAMOTO, Shonan University of Medical Sciences, JAPAN, Japan, Email: chisako.yamamoto@sums.ac.jp

TANJI HOSHI, Tokyo Metropolitan University, Japan, Email: star@onyx.dti.ne.jp

\section{Citation:}

CHISAKO YAMAMOTO, TANJI HOSHI (2018). Lifestyle and dietary factors associating with dementia status in the community-dwelling elderly aged 65 and older in a suburban town of Tokyo. International Journal of Social Sciences, Vol. VII(2), pp. 109-133., 10.20472/SS.2018.7.2.006 


\section{Introduction}

The World Health Organization (WHO) and Alzheimer's Disease International (ADI) jointly reported "Dementia: a public health priority" in 2012 to raise awareness of dementia as a public health priority, to articulate a public health approach and to advocate for action at international and national levels. The United Kingdom hosted the G8 Dementia Summit for the first time in London at the occasion of hosting the G8 Summit in December 2013. The invited Health Ministers discussed how to shape an effective international response to dementia (G8 DEMENTIA SUMMIT DECLARATION, 2013). In May 2016 Japan hosted G7 Ise-Shima Summit and the G7 leaders announced to commit to take concrete actions articulated in G7 Ise-Shima Vision for Global Health, in which they were to pursue supporting for communities to become dementia-friendly, including Age and Dementiafriendly Communities, and promoting Dementia Supporters/Friends (G7 Ise-Shima Vision for Global Health, 2016). Building dementia-friendly communities was discussed at the $32^{\text {nd }}$ International Conference of Alzheimer's Disease International in April 2017 and followed in May by the adoption of Global Action Plan on the Public Health Response to Dementia by the $70^{\text {th }}$ World Health Assembly (WHO).

Older people constitute an increasing proportion of total population, as the rise in life expectancy is being accompanied by declining fertility rates in most countries. It is estimated that 46.8 million people worldwide were living with dementia in 2015 . This number will almost double every 20 years, reaching 74.7 million in 2030 and 131.5 million in 2050 (Alzheimer's Disease International, 2015).

The successes of improved health care in high income countries have increased the number of the elderly. Many of them are living longer than before. Japan has been enjoying great longevity, whose life expectancy at birth was 80.98 in men and 87.14 in women in 2016. It will further extend to 84.19 and 90.93 in men and women, respectively, in 2060 (Ministry of Health, Labour and Welfare, 2017). The elderly rate was $27.3 \%$ as of October 1,2016 and is estimated to rise to $39.9 \%$ in 2060 (Cabinet Office, 2016). Asada et al. (2013) projected that the number of people with dementia was 4.62 million in 2012 and the prevalence of dementia of the elderly aged 65 and older was $15 \%$ and the following research projected people with dementia will be 7 million and the prevalence $20.9 \%$ in 2025 in Japan.

Since early detection and implementation of treatment are essential, the Japanese government initiated more effective measures to make dementia friendly communities and implemented the notable program entitled "Nationwide Caravan to Train One Million Dementia Supporters" in 2005. Under these measures, municipalities have been providing lectures on preventing dementia, promoting understanding of early symptoms and training dementia supporters in communities over the past years. They attained the initial goal of one million dementia supporters in May 2009. At the end of March 2017, the number of supporters reached 8.83 million (Dementia Supporters Caravan, 2017) and is planned to increase up to 10 million by 2025 . 
Speaking of addressing with the issue of dementia before 2005, Japanese local governments conducted forty-five sample surveys from 1956 to 2001 on the prevalence of dementia. The prevalence ranged from $3.0 \%$ to $8.8 \%$. The number of subjects varied from 497 in Miyagi Tajiri Town in the eastern part of Japan in 1998 to 9,274 in Hokkaido in the northern part in 1986. Tokyo metropolis, which has a population of 13.65 million as in December 2017, conducted surveys in 1973, 1980, 1988 and 1995. Prevalence of dementia was $4.5 \%, 4.6 \%, 4.0 \%$ and $4.1 \%$, respectively. Toyama Prefecture, which has a population of 1.08 million as in January 2016 and is located in the middle of Japan, facing to the Japan Sea, conducted surveys in 1982, 1985, 1990, 1996 and 2001. Prevalence was $5.6 \%, 4.5 \%, 5.7 \%, 4.5 \%$ and $8.8 \%$, respectively (Nakamura S, Shigeta M, Iwamoto $\mathrm{M}$, et al., 2004). Wide range of prevalence could be partly due to the methods of assessment, including diagnostic criteria used. Many of previous reports in Japan showed prevalence around $5.0 \%$ and no particular tendency of increasing or decreasing could be observed. It might be partly because there were not many programs and measures against dementia initiated by municipalities before 2001. Yamamoto and Hoshi (2012) reported that the prevalence of dementia in City A, which is in the suburbs of Tokyo metropolis, is steadily decreasing during a 6 -year follow-up from $9.7 \%$ in $2001,7.8 \%$ in 2004 and to $7.4 \%$ in 2007 . Results are based on the complete surveys of the community-dwelling elderly of 65 years and older. The number of analysis subjects was 13,058 in 2001, 13,182 in 2004 and 15,084 in 2007 . Some may simply argue that it is due to institutionalization of persons with dementia status. However, institutionalized ones are excluded from the subject elderly in their study. Complete surveys reflect the real state of the population more exactly than sample surveys. It is essential for analytical studies to focus on gender differences to provide useful knowledge, since statistical figures differ by gender, the best example of which is average life expectancy.

'Nutrition and Dementia' published by Alzheimer's Disease International in 2014 reports the undernutrition and weight loss among people with dementia. An unhealthy lifestyle and diet are likely to cause many diseases. Since Japan enjoys great longevity as described above, it is worth to review Japanese elderly lifestyle and diet and to examine the association with their dementia status.

The purpose of this study is two-folds: to clarify lifestyle and dietary factors associating with dementia status in the community-dwelling elderly aged 65 and older in Town $\mathrm{B}$, a suburban town of Tokyo, and to clarify gender differences in health behavior.

The remainder of this article is organized as follows. In the next section methods are introduced, in which data collection, study population, analytical methods and ethical considerations are described. In the third section results and findings are shown and their implications are discussed in the fourth section. 


\section{Methods}

\subsection{Outline of Study Field}

Town B is located in the suburbs of Shinjuku, a sub-center of Tokyo, Japan, at a distance of 60-70 minutes' train ride. It has natural beauty surrounded by mountains and lakes and is a small town whose population in 2005 was 10,823 . It was absorbed into the neighboring city in 2007.

\subsection{Data Collection}

Data were collected in February 2004. Self-administered questionnaires were mailed to 2,069 community-dwelling elderly people aged 65 and older. Institutionalized ones were excluded. Questionnaires consisted of 56 items such as fundamental attributes, respondents (addressee himself or herself, or proxy), family members, self-perceived health, body parts feeling pains, diseases under treatment, dietary items, smoking, alcohol drinking, activities of daily living, instrumental activities of daily living, lifestyles, leisure activities, occupations, life satisfaction, socio-economic status and others. The number of responses returned was 1,538 (response rate; $74.3 \%$ ). They were returned by mail by addressees themselves or proxies when the addressees were unable to respond for some reasons such as absence, being hospitalized, cognitive impairment or dementia status.

\subsection{Analysis Subjects}

After inappropriate responses were excluded, the number of analysis subjects was 1,436 (649 men and 787 women), among which 1,338 addressees (616 men and 722 women) responded by themselves and 187 by proxies (65 men and 122 women) who were all family caregivers. The number of analysis subjects by age groups is shown in Table 1. Mean age (sd: standard deviation) was 74.2 (6.6) in men and 75.6 (7.2) in women.

\begin{tabular}{|c|c|c|c|c|c|c|c|}
\hline & & (65-69 & $70-74$ & $75-79$ & (80-84 & $85+$ & Total \\
\hline \multirow[t]{4}{*}{ Men } & & 203(31.3) & $157(24.2)$ & $154(23.7)$ & $85(13.1)$ & $50(7.7)$ & $649(100.0)$ \\
\hline & PWD & $0(0.0)$ & $0(0.0)$ & $5(31.3)$ & $5(31.3)$ & $6(37.5)$ & $16(100.0)$ \\
\hline & PPD & $11(21.2)$ & $9(17.3)$ & $10(19.2)$ & $11(21.2)$ & $11(21.2)$ & $52(100.0)$ \\
\hline & CIP & 192(33.0) & 148(25.5) & $139(23.9)$ & $69(11.9)$ & $33(5.7)$ & $581(100.0)$ \\
\hline \multirow[t]{4}{*}{ Women } & & 182(23.2) & $211(26.8)$ & $176(22.4)$ & 116(14.7) & 102(13.0) & $787(100.0)$ \\
\hline & PWD & $1(2.8)$ & $6(16.7)$ & $4(11.1)$ & $8(22.2)$ & $17(47.2)$ & $36(100.0)$ \\
\hline & PPD & $8(6.6)$ & $19(15.7)$ & $29(24.0)$ & $21(17.4)$ & $44(36.4)$ & $121(100.0)$ \\
\hline & CIP & $173(27.5)$ & 186(29.5) & $143(22.7)$ & $87(13.8)$ & $41(6.5)$ & $630(100.0)$ \\
\hline
\end{tabular}

(Note) PWD: People with Dementia, PPD: People with Probable Dementia, CIP: Cognitively Intact People.

Among analysis subjects, people whose proxies returned responses due to cognitive impairment or dementia were defined as people with dementia (PWD). Precisely speaking they were not diagnosed but recognized and judged to have dementia status by family caregivers. Yamamoto and Hoshi examined cognitive status of people who returned responses by themselves in the population-based complete survey of a suburban city of 
Tokyo (Yamamoto C and Hoshi T, 2010). They reported there were people whose dementia status was recognized neither by themselves nor by family members but overlooked. In their study overlooked dementia was measured by three cognitive capacities: handling one's own banking, filling out forms for pension, and reading books and/or newspapers, which are based on Tokyo Metropolitan Institute of Gerontology Index of Competence (TMIG-Index of competence) (Koyano W, Shibata H, Nakazato K et al. , 1987) (See No.28 in Appendix) with a partial modification. One point was allocated to positive answers, and the cut-off point determined by receiver operator characteristic curves was $0-1 / 2-3$. Their results suggested that the proportion of the $0-1$ scoring group was the one whose dementia status was overlooked in association with mortality and longevity, although they themselves and family members regarded them healthy without dementia status. We applied the same methods to this study. We define the 0-1 scoring group as people with probable dementia (PPD) and the 2-3 scoring group as cognitively intact people (CIP).

The number of analysis subjects grouped by cognitive scores is also shown in Table 1 . Mean age (sd) was 82.7 (5.2) in men and 82.8 (7.3) in women in PWD, 77.6 (7.5) in men and 81.2 (7.9) in women in PPD, and 73.5 (6.2) in men and 74.1 (6.3) in women in CIP, respectively.

\subsection{Measurement}

\subsubsection{Questionnaire Items and Scoring of Frequency}

Questionnaire items used in this study were weight and height to calculate body mass index (BMI) $\left[\mathrm{kg} / \mathrm{m}^{2}\right]$, the number of natural teeth in total and nine lifestyle and 13 dietary items. Nine lifestyle items were smoking, alcohol drinking, cooking, breakfast eating, walking/exercise, sleep duration per day including naps, going out, daytime lying duration in bed and the number of hobbies, and 13 dietary items were meat/poultry, soybean products, eggs, oily fish, dairy products, fruits, vegetables, salted fish such as salmon and cod and dried fish, heavily seasoned food, deep-/stir-fried food, miso soup/soup, pickled ume plums and pickled vegetables.

The number of natural teeth was classified into five groups: 0. no teeth, 1. 1-9 teeth, 2. 1019 teeth, 3. 20-28 teeth, and 4. 29-32 teeth. The Three-point and Four-point Scales were used for scoring frequency of lifestyle and dietary items. Although all the questionnaire items and scales are shown in Appendix, for convenience, nine lifestyle items and scales are as follows: 1) Smoking: 1. never smoked, 2. quitted, and 3. still smoking. 2) Alcohol drinking: 1. rarely, 2. once or twice per week, 3. 3-5 times per week, and 4. almost every day. 3) Cooking: 1. often, 2. sometimes, and 3. rarely. 4) Breakfast eating: 1. almost every day, 2. sometimes, 3. rarely, and 4. never. 5) Walking/exercise: 1. almost every day, 2. 35 times per week, 3. once or twice per week, and 4. rarely. 6) Sleep duration per day including naps: 1 . less than 6 hours, 2. 7-8 hours, and 3. more than 9 hours. 7) Going out: 1. almost every day, 2. 3-4 times per week, 3. once or twice per week, and 4. less than once per month. 8) Daytime lying duration in bed: 1. out of bed, 2. out of bed longer than 
in bed, 3. in bed longer than out of bed, and 4. almost in bed, and 9) The number of hobbies: 1. more than two, 2. just one, and 3. none. The cross tabulation of lifestyle items by dementia status is shown in Table 2 .

Ten out of 13 dietary items, i.e., 1) Meat/poultry, 2) soybean products such as tofu (soybean curd) and natto (fermented soybeans) , 3) Eggs, 4) Oily fish, 5) Dairy products, 6) Fruits, 7) Vegetables, 8) Salted fish such as salmon and cod and dried fish, 9) Heavily seasoned food, 10) Deep- or stir-fried food were measured by the Four-point Scale: 1. almost every day, 2. sometimes, 3 . rarely, and 4 . never. The remaining dietary items and their scales are as follows: 11) Miso (fermented soybean paste) soup/soup per day: 1. more than 3 bowls, 2 . 2 bowls, 3. 1 bowl, and 4. no bowls. 12) The number of pickled ume plums per day: 1. more than three, 2. two, and 3. one, and 4. none. 13) The frequency of pickled vegetables per day: 1 . more than 3 times, 2 . twice, 3. once, and 4. never. The cross tabulation of dietary items by dementia status is shown in Table 3.

Table 2. Lifestyle items and proportion of the elderly by dementia status

\begin{tabular}{|c|c|c|c|c|c|c|c|}
\hline & & \multicolumn{3}{|c|}{ Men } & \multicolumn{3}{|c|}{ Women } \\
\hline & & PWD $n(\%)$ & PPD $n(\%)$ & $\operatorname{CIP} \mathrm{n}(\%)$ & PWD n(\%) & PPD $n(\%)$ & CIP $n(\%)$ \\
\hline \multirow[t]{3}{*}{ Smoking } & 1. never smoked & $4(28.6)$ & $19(38.8)$ & $152(28.5)$ & $25(92.6)$ & $98(86.7)$ & $516(91.5)$ \\
\hline & 2. quitted & $8(57.1)$ & $21(42.9)$ & $254(47.6)$ & $1(3.7)$ & $13(11.5)$ & $23(4.1)$ \\
\hline & 3. still smoking & $2(14.3)$ & $9(18.4)$ & $128(24.0)$ & $1(3.7)$ & $2(1.8)$ & $25(4.4)$ \\
\hline \multirow[t]{4}{*}{ Alcohol drinking } & 1. rarely & $13(92.9)$ & $37(75.5)$ & $243(44.8)$ & $29(96.7)$ & $108(95.6)$ & $494(88.5)$ \\
\hline & 2. once or twice/w & $1(7.1)$ & $3(6.1)$ & $53(9.8)$ & $1(3.3)$ & $2(1.8)$ & $34(6.1)$ \\
\hline & 3. 3-5 times $/ \mathrm{w}$ & $0(0.0)$ & $1(2.0)$ & $55(10.1)$ & $0(0.0)$ & $0(0.0)$ & $12(2.2)$ \\
\hline & 4. almost every day & $0(0.0)$ & $8(16.3)$ & $192(35.4)$ & $0(0.0)$ & $3(2.7)$ & $18(3.2)$ \\
\hline \multirow[t]{3}{*}{ Cooking } & 1. often & $0(0.0)$ & $5(10.2)$ & $78(14.4)$ & $0(0.0)$ & $27(23.5)$ & $480(75.2)$ \\
\hline & 2. sometimes & $0(0.0)$ & $1(2.0)$ & $109(20.1)$ & $1(3.4)$ & $17(14.8)$ & $110(18.0)$ \\
\hline & 3. rarely & $13(100.0)$ & $43(87.8)$ & $354(65.4)$ & $28(96.6)$ & $71(61.7)$ & $42(6.9)$ \\
\hline \multirow[t]{4}{*}{ Breaskfast eating } & 1. almost every day & $13(100.0)$ & $42(95.5)$ & $521(94.7)$ & $25(86.2)$ & $100(89.3)$ & $560(93.6)$ \\
\hline & 2. sometimes & $0(0.0)$ & $0(0.0)$ & $12(2.2)$ & $3(10.3)$ & $8(7.1)$ & $26(4.3)$ \\
\hline & 3. rarely & $0(0.0)$ & $2(4.5)$ & $8(1.5)$ & $1(3.4)$ & $1(0.9)$ & $9(1.5)$ \\
\hline & 4. never & $0(0.0)$ & $0(0.0)$ & $9(1.6)$ & $0(0.0)$ & $3(2.7)$ & $3(0.5)$ \\
\hline \multirow[t]{4}{*}{ Walking/exercise } & 1. almost every day & $0(0.0)$ & $15(34.1)$ & $229(43.2)$ & $8(30.8)$ & $31(29.0)$ & $212(36.7)$ \\
\hline & 2. $3-5$ times/w & $5(41.7)$ & $6(13.6)$ & $96(18.1)$ & $3(11.5)$ & $17(15.9)$ & $135(23.4)$ \\
\hline & 3. once or twice/w & $5(41.7)$ & $7(15.9)$ & $111(20.9)$ & $10(38.5)$ & $31(29.0)$ & $173(30.0)$ \\
\hline & 4. rarely & $2(16.7)$ & $16(36.4)$ & $94(17.7)$ & $5(19.2)$ & $28(26.2)$ & $57(9.9)$ \\
\hline \multirow{3}{*}{$\begin{array}{l}\text { Sleep duration/d } \\
\text { including naps }\end{array}$} & 1. less than $6 \mathrm{hrs}$ & $1(7.1)$ & $6(12.2)$ & $88(15.9)$ & $3(10.0)$ & $14(12.1)$ & $134(22.0)$ \\
\hline & 2. $7-8 \mathrm{hrs}$. & $5(35.7)$ & $18(36.7)$ & $347(62.7)$ & $5(16.7)$ & $51(44.0)$ & $384(63.0)$ \\
\hline & 3. more than $9 \mathrm{hrs}$ & $8(57.1)$ & $25(51.0)$ & $118(21.3)$ & $22(73.3)$ & $51(44.0)$ & $92(15.1)$ \\
\hline \multirow[t]{4}{*}{ Going out } & 1. almost every day & $0(0.0)$ & $6(13.6)$ & $199(35.5)$ & $3(11.1)$ & $9(8.5)$ & $100(17.0)$ \\
\hline & 2. 3-4 times/w & $6(60.0)$ & $6(13.6)$ & $120(21.4)$ & $3(11.1)$ & $8(7.5)$ & $138(23.4)$ \\
\hline & 3. once or twice/w & $1(10.0)$ & $14(31.8)$ & $188(33.5)$ & $6(22.2)$ & $44(41.5)$ & $263(44.7)$ \\
\hline & 4. less than once/m & $3(30.0)$ & $18(40.9)$ & $54(9.6)$ & $15(55.6)$ & $45(42.5)$ & $88(14.9)$ \\
\hline \multirow{4}{*}{$\begin{array}{l}\text { Daytime lying } \\
\text { duration in bed }\end{array}$} & 1. almost out of bed & $7(46.7)$ & $21(43.8)$ & $449(81.2)$ & $10(31.3)$ & $60(52.6)$ & $488(83.4)$ \\
\hline & 2. out of bed longer than in bed & $5(33.3)$ & $9(18.8)$ & $75(13.6)$ & $12(37.5)$ & $25(21.9)$ & $80(13.7)$ \\
\hline & 3. in bed longer than out of bed & $3(20.0)$ & $9(18.8)$ & $6(1.1)$ & $5(15.6)$ & $16(14.0)$ & $7(1.2)$ \\
\hline & 4. almost in bed & $0(0.0)$ & $9(18.8)$ & $23(4.2)$ & $5(15.6)$ & $13(11.4)$ & $10(1.7)$ \\
\hline \multirow[t]{3}{*}{ Hobbies } & 1. more than two & $0(0.0)$ & $7(14.3)$ & $283(51.6)$ & $4(15.4)$ & $20(17.9)$ & $248(42.8)$ \\
\hline & 2. just one & $3(25.0)$ & $10(20.4)$ & $157(28.6)$ & $1(3.8)$ & $29(25.9)$ & $196(33.8)$ \\
\hline & 3. none & $9(75.0)$ & $32(65.3)$ & $108(19.7)$ & $21(80.8)$ & $63(56.3)$ & $136(23.4)$ \\
\hline
\end{tabular}

(Note) The number of missed responses was not included in parameters. 


\subsubsection{Analytical Methods}

Descriptive statistics, a chi-square, Kruskal-Wallis and Mann-Whitney $U$ tests and Bonferroni's multiple comparisons were performed in men and women, respectively. Since the data do not follow the normal distribution, Kruskal-Wallis test was performed for ordinal scales to determine median $\left(25^{\text {th }}-75^{\text {th }}\right.$ percentile $)$. Using Mann-Whitney $U$ test, Bonferroni's multiple comparisons as post hoc analyses were performed to find significant differences between three groups.

Significance was set at $0.05(0.05 / 3=0.017,0.01 / 3=0.003$ and $0.001 / 3=0.0003$ after Bonferroni correction). Data were analyzed by using SPSS11.0J for Windows. 
Table 3. Dietary items and proportion of the elderly by dementia status

\begin{tabular}{|c|c|c|c|c|c|c|c|}
\hline & & \multicolumn{3}{|c|}{ Men } & \multicolumn{3}{|c|}{ Women } \\
\hline & & PWD $\mathrm{n}(\%)$ & PPD n(\%) & CIP $n(\%)$ & PWD n(\%) & PPD n(\%) & CIP $n(\%)$ \\
\hline \multirow[t]{4}{*}{ Meat/poultry } & 1. almost every day & $1(7.7)$ & $3(6.4)$ & $55(10.3)$ & $3(10.0)$ & $4(3.8)$ & $68(11.9)$ \\
\hline & 2. sometimes & $8(61.5)$ & $32(68.1)$ & $380(71.2)$ & $20(66.7)$ & $64(61.5)$ & $402(70.2)$ \\
\hline & 3. rarely & $3(23.1)$ & $11(23.4)$ & $94(17.6)$ & $6(20.0)$ & $26(25.0)$ & $92(16.1)$ \\
\hline & 4. never & $1(7.7)$ & $1(2.1)$ & $5(0.9)$ & $1(3.3)$ & $10(9.6)$ & $11(1.9)$ \\
\hline \multirow[t]{4}{*}{ Soybean products } & 1. almost every day & $7(53.8)$ & $21(45.7)$ & $263(48.9)$ & $7(22.6)$ & $38(34.9)$ & $327(55.2)$ \\
\hline & 2. sometimes & $6(46.2)$ & $21(45.7)$ & $257(47.8)$ & $22(71.0$ & $54(58.7)$ & $254(42.9)$ \\
\hline & 3. rarely & $0(0.0)$ & $3(6.5)$ & $15(2.8)$ & $2(6.5)$ & $4(3.7)$ & $10(1.7)$ \\
\hline & 4. never & $0(0.0)$ & $1(2.2)$ & $3(0.6)$ & $0(0.0)$ & $3(2.8)$ & $1(0.2)$ \\
\hline \multirow[t]{4}{*}{ Eggs } & 1. almost every day & $3(23.1)$ & $17(37.0)$ & $174(32.5)$ & $10(35.7)$ & $25(23.1)$ & $219(37.8)$ \\
\hline & 2. sometimes & $10(76.9)$ & $24(52.2)$ & $294(55.0)$ & $18(64.3)$ & $73(67.6)$ & $294(50.7)$ \\
\hline & 3. rarely & $0(0.0)$ & $5(10.9)$ & $60(11.2)$ & $0(0.0)$ & $8(7.4)$ & $62(10.7)$ \\
\hline & 4. never & $0(0.0)$ & $0(0.0)$ & $7(1.3)$ & $0(0.0)$ & $2(1.9)$ & $5(0.9)$ \\
\hline \multirow[t]{4}{*}{ Oily fish } & 1. almost every day & $1(7.7)$ & $7(15.6)$ & $90(16.7)$ & $4(13.3)$ & $14(13.0)$ & $98(15.4)$ \\
\hline & 2. sometimes & $10(76.9)$ & $28(62.2)$ & $389(72.3)$ & $20(66.7)$ & $77(71.3)$ & $427(71.6)$ \\
\hline & 3. rarely & $1(7.7)$ & $7(15.6)$ & $51(9.5)$ & $4(13.3)$ & $12(11.1)$ & $59(9.9)$ \\
\hline & 4. never & $1(7.7)$ & $3(6.7)$ & $8(1.5)$ & $2(6.7)$ & $5(4.6)$ & $12(2.0)$ \\
\hline \multirow[t]{4}{*}{ Dairy products } & 1. almost every day & $7(53.8)$ & $19(43.2)$ & $236(44.5)$ & $12(40.0)$ & $46(42.2)$ & $327(55.3)$ \\
\hline & 2. sometimes & $2(15.4)$ & $15(34.1)$ & $195(36.8)$ & $13(43.3)$ & $38(34.9)$ & $171(28.9)$ \\
\hline & 3. rarely & $2(15.4)$ & $5(11.4)$ & $74(14.0)$ & $4(13.3)$ & $14(12.8)$ & $70(11.8)$ \\
\hline & 4. never & $2(15.4)$ & $5(11.4)$ & $25(4.7)$ & $1(3.3)$ & $11(10.1)$ & $23(3.9)$ \\
\hline \multirow{4}{*}{ Fruits } & 1. almost every day & $4(30.8)$ & $15(31.9)$ & $254(47.3)$ & $14(48.3)$ & $36(32.1)$ & $387(65.6)$ \\
\hline & 2. sometimes & $8(61.5)$ & $25(53.2)$ & $228(42.5)$ & $15(51.7)$ & $60(53.6)$ & $177(30.0)$ \\
\hline & 3. rarely & $1(7.7)$ & $6(12.8)$ & $48(8.9)$ & $0(0.0)$ & $14(12.5)$ & $25(4.2)$ \\
\hline & 4. never & $0(0.0)$ & $1(2.1)$ & $7(1.3)$ & $0(0.0)$ & $2(1.8)$ & $1(0.2)$ \\
\hline \multirow[t]{4}{*}{ Vegetables } & 1. almost every day & $8(61.5)$ & $31(68.9)$ & $374(71.1)$ & $16(51.6)$ & $71(65.1)$ & $504(87.5)$ \\
\hline & 2. sometimes & $5(36.5)$ & $12(26.7)$ & $137(26.0)$ & $14(45.2)$ & $32(29.4)$ & $70(2.2)$ \\
\hline & 3. rarely & $0(0.0)$ & $2(4.4)$ & $13(2.5)$ & $1(3.2)$ & $3(2.8)$ & $2(0.3)$ \\
\hline & 4. never & $0(0.0)$ & $0(0.0)$ & $2(0.4)$ & $0(0.0)$ & $3(2.8)$ & $0(0.0)$ \\
\hline \multirow[t]{4}{*}{ Salted fish } & 1. almost every day & $1(12.5)$ & $1(3.2)$ & $41(11.3)$ & $3(21.4)$ & $7(9.9)$ & $57(11.89$ \\
\hline & 2. sometimes & $5(62.5)$ & $21(67.7)$ & $214(59.0)$ & $7(50.0)$ & $40(56.3)$ & $292(60.2)$ \\
\hline & 3. rarely & $1(12.5)$ & $5(16.1)$ & $94(25.9)$ & $3(21.4)$ & $22(31.0)$ & $122(25.2)$ \\
\hline & 4. never & $1(12.5)$ & $4(12.9)$ & $14(3.9)$ & $1(7.1)$ & $2(2.8)$ & $14(2.9)$ \\
\hline \multirow{4}{*}{$\begin{array}{l}\text { Heavily seasoned } \\
\text { food }\end{array}$} & 1. almost every day & $0(0.0)$ & $6(13.0)$ & $69(13.2)$ & $3(10.3)$ & $12(11.0)$ & $70(12.3)$ \\
\hline & 2. sometimes & $5(41.7)$ & $20(43.5)$ & $193(36.9)$ & $17(58.6)$ & $39(35.8)$ & $180(31.6)$ \\
\hline & 3. rarely & $7(58.3)$ & $13(28.3)$ & $223(42.6)$ & $5(17.2)$ & $43(39.4)$ & $273(47.9)$ \\
\hline & 4. never & $0(0.0)$ & $7(15.2)$ & $38(7.3)$ & $4(13.8)$ & $15(13.8)$ & $47(8.2)$ \\
\hline \multirow{4}{*}{$\begin{array}{l}\text { Deep-/stir-fried } \\
\text { food }\end{array}$} & 1. almost every day & $0(0.0)$ & $5(11.1)$ & $62(11.6)$ & $1(3.4)$ & $9(8.3)$ & $102(17.2)$ \\
\hline & 2. sometimes & $8(61.5)$ & $28(62.2)$ & $368(69.0)$ & $21(72.4)$ & $73(67.0)$ & $387(65.4)$ \\
\hline & 3. rarely & $5(38.5)$ & $11(24.4)$ & $99(18.6)$ & $6(20.7)$ & $22(20.2)$ & $96(16.2)$ \\
\hline & 4. never & $0(0.0)$ & $1(2.2)$ & $4(0.8)$ & $1(3.4)$ & $5(4.6)$ & $7(1.2)$ \\
\hline \multirow[t]{4}{*}{ Miso soup/soup/d } & 1. more than 3 bowls & $3(21.4)$ & $8(16.7)$ & $88(16.0)$ & $8(27.6)$ & $28(24.8)$ & $61(10.1)$ \\
\hline & 2. two bowls & $3(21.4)$ & $18(37.5)$ & $189(34.3)$ & $10(34.5)$ & $29(25.7)$ & $215(35.6)$ \\
\hline & 3. one bowl & $8(57.1)$ & $18(37.5)$ & $255(46.3)$ & $9(31.0)$ & $47(41.6)$ & $303(50.2)$ \\
\hline & 4. no bowls & $0(0.0)$ & $4(8.3)$ & $19(3.4)$ & $2(6.9)$ & $9(8.0)$ & $25(4.1)$ \\
\hline \multirow{4}{*}{$\begin{array}{l}\text { Nr. of pickled ume } \\
\text { plums } / d\end{array}$} & 1. more than three & $0(0.0)$ & $1(2.2)$ & $17(3.1)$ & $2(6.9)$ & $2(1.8)$ & $21(3.6)$ \\
\hline & 2. two & $0(0.0)$ & $2(4.4)$ & $48(8.9)$ & $0(0.0)$ & $12(10.8)$ & $64(11.0)$ \\
\hline & 3. one & $9(69.2)$ & $18(40.0)$ & $236(43.6)$ & $15(51.7)$ & $52(46.8)$ & $294(50.4)$ \\
\hline & 4. none & $4(30.8)$ & $24(53.3)$ & $240(44.4)$ & $12(41.4)$ & $45(40.5)$ & $204(35.0)$ \\
\hline \multirow{4}{*}{$\begin{array}{l}\text { Pickled vegeta- } \\
\text { bles/d }\end{array}$} & 1. more than 3 times & $0(0.0)$ & $7(14.3)$ & $140(25.3)$ & $4(13.3)$ & $19(16.7)$ & $172(28.3)$ \\
\hline & 2. twice & $3(21.4)$ & $15(30.6)$ & $173(31.3)$ & $4(13.3)$ & $31(27.2)$ & $224(36.9)$ \\
\hline & 3. once & $6(42.9)$ & $13(26.5)$ & $158(28.6)$ & $12(40.0)$ & $36(31.6)$ & $159(26.2)$ \\
\hline & 4. never & $5(35.7)$ & $14(28.6)$ & $82(14.8)$ & $10(33.3)$ & $28(24.6)$ & $52(8.6)$ \\
\hline
\end{tabular}

(Note) The number of missed responses was not included in parameters. 


\subsubsection{Ethical Considerations}

Tokyo Metropolitan University made an agreement with Town B to protect personal data. The University Committee on Ethical Issues approved the surveys and the study. Individuals were all numbered without names, and an alternative "I don't want to answer." option was provided in some items. We assumed all respondents (and proxies) who returned the questionnaires to have consented to the survey.

\section{Results}

\subsection{Attributes of analysis subjects by dementia status}

Mean weight and BMI are shown in Table 4. Mean age is reshown to clarify the characteristics of dementia status. PWD was the oldest and CIP the youngest in men and women, respectively. Mean weight and BMI were the smallest in PWD and the largest in CIP in both men and women. Thus, PWD was the oldest and thinnest in men and women, respectively.

Table 4. Attributes of analysis subjects

\begin{tabular}{|c|c|c|c|c|c|}
\hline & Dementia status & $\mathrm{n}(\%)$ & $\begin{array}{l}\text { Mean age } \\
\text { (sd) }\end{array}$ & $\begin{array}{c}\text { Mean weight }(\mathrm{sd}) \\
\text { in } \mathrm{kg}\end{array}$ & BMI（sd） \\
\hline \multirow[t]{3}{*}{ Men } & PWD & $16(2.5)$ & $82.7(5.2)$ & $53.3(6.4)$ & $21.9(2.0)$ \\
\hline & PPD & $52(8.0)$ & $77.6(7.5)$ & $57.3(8.6)$ & $22.1(2.8)$ \\
\hline & CIP & $581(89.5)$ & $73.5(6.2)$ & $61.0(11.6)$ & $23.2(4.3)$ \\
\hline \multirow[t]{3}{*}{ Women } & PWD & $36(4.6)$ & $82.8(7.3)$ & $44.1(6.0)$ & $20.9(2.8)$ \\
\hline & PPD & $121(15.4)$ & $81.2(7.9)$ & $47.9(9.0)$ & $22.4(3.8)$ \\
\hline & CIP & $630(80.0)$ & $74.1(6.3)$ & $51.5(9.2)$ & $23.1(4.1)$ \\
\hline
\end{tabular}

(Note) The number of analysis subjects: 1,436 ( 649 men and 787 women).

PWD: People with Dementia, PPD: People with Probable Dementia, CIP: Cognitively Intact People.

\subsection{Results of Kruskal-Wallis and $x^{2}$ tests and Bonferroni's multiple comparisons}

Mann-Whitney $U$ test was used to find out if there was a significant difference between two groups, i.e., CIP and PPD, PPD and PWD, and PWD and CIP. Results in men and women are shown in Table 5.

\subsubsection{Association between lifestyles and dementia status}

Significant differences were shown in alcohol drinking, cooking, walking/exercise, sleep duration per day including naps, going out, daytime lying duration in bed and the number of hobbies in both men and women. Those in smoking and breakfast eating were shown neither in men nor in women.

As for dietary items in women, significant differences were shown in nine items, which were meat/poultry, soybean products, oily fish, dairy products, fruits, vegetables, deep/stir-fried food, miso soup/soup and pickled vegetables, however, a significant difference in men was shown only in pickled vegetables. 
Table 5. Results of Kruskal-Wallis and $\chi 2$ tests and Bonferroni's multiple comparisons in men and

\begin{tabular}{|c|c|c|c|c|c|}
\hline & Lifestile \& Dietary items & $\begin{array}{l}\text { Median(25th-75th } \\
\text { percentile) } \\
\text { a.b. } \\
\end{array}$ & $\begin{array}{c}\chi^{2} \\
(\mathrm{df}=2)\end{array}$ & $p$-value & $\begin{array}{c}\text { Bonferroni's multiple } \\
\text { comparisons }\end{array}$ \\
\hline \multirow[t]{22}{*}{ Men } & Nr. of natural teeth & $2.00(1.00-3.00)$ & 6.97 & 0.031 & $\mathrm{PPD}<\mathrm{CIP}^{*}$ \\
\hline & Smoking & $2.00(1.00-2.00)$ & 2.20 & 0.332 & - \\
\hline & Alcohol drinking & $2.00(1.00-4.00)$ & 26.51 & $<0.001$ & $\mathrm{PPD}<\mathrm{CIP}^{* * *} \quad \mathrm{PWD}<\mathrm{CIP}^{* * *}$ \\
\hline & Cooking & $3.00(2.00-3.00)$ & 14.45 & 0.001 & $\mathrm{PPD}<\mathrm{CIP}^{* *} \quad \mathrm{PWD}<\mathrm{CIP}^{*}$ \\
\hline & Breakfast eating & $1.00(1.00-1.00)$ & 0.76 & 0.686 & - \\
\hline & Walking/exercise & $2.00(1.00-3.00)$ & 8.41 & 0.015 & $\mathrm{PPD}<\mathrm{CIP}^{*} \quad \mathrm{PWD}<\mathrm{CIP}^{*}$ \\
\hline & Sleep duration/d & $2.00(2.00-3.00)$ & 21.12 & $<0.001$ & $\mathrm{PPD}<\mathrm{CIP}^{* * *} \mathrm{PWD}<\mathrm{CIP}^{*}$ \\
\hline & Going out & $2.00(1.00-3.00)$ & 25.64 & $<0.001$ & $\mathrm{PPD}<\mathrm{CIP}^{* * *}$ \\
\hline & Daytime lying duration & $1.00(1.00-1.00)$ & 49.89 & $<0.001$ & $\mathrm{PPD}<\mathrm{CIP}^{* * *} \mathrm{PWD}<\mathrm{CIP}^{* *}$ \\
\hline & Nr. of hobbies & $2.00(1.00-2.00)$ & 59.45 & $<0.001$ & $\mathrm{PPD}<\mathrm{CIP}^{* * *} \quad \mathrm{PWD}<\mathrm{CIP}^{* * *}$ \\
\hline & Meat/poultry & $2.00(2.00-2.00)$ & 2.95 & 0.229 & - \\
\hline & Soybean products & $2.00(1.00-2.00)$ & 0.80 & 0.670 & - \\
\hline & Eggs & $2.00(1.00-2.00)$ & 0.42 & 0.809 & - \\
\hline & Oily fish & $2.00(2.00-2.00)$ & 3.13 & 0.210 & - \\
\hline & Dairy products & $2.00(1.00-2.00)$ & 0.27 & 0.873 & - \\
\hline & Fruits & $2.00(1.00-2.00)$ & 4.87 & 0.088 & - \\
\hline & Salted fish & $2.00(2.00-3.00)$ & 0.69 & 0.707 & - \\
\hline & Heavily seasoned food & $2.00(2.00-3.00)$ & 0.41 & 0.816 & - \\
\hline & Deep-/stir-fried food & $2.00(2.00-2.00)$ & 4.60 & 0.100 & - \\
\hline & Miso soup/soup & $2.00(2.00-3.00)$ & 0.03 & 0.986 & 一 \\
\hline & Pickled ume plums & $3.00(3.00-4.00)$ & 1.96 & 0.376 & - \\
\hline & Pickleld vegetables & $2.00(2.00-3.00)$ & 13.45 & 0.001 & $\mathrm{PPD}<\mathrm{CIP}^{*} \mathrm{PWD}<\mathrm{CIP}^{* *}$ \\
\hline \multirow[t]{23}{*}{ Women } & Nr. of natural teeth & $2.00(1.00-3.00)$ & 40.74 & $<0.001$ & $\mathrm{PPD}<\mathrm{CIP}^{* * *} \mathrm{PWD}<\mathrm{CIP}^{* * *}$ \\
\hline & Smoking & $1.00(1.00-1.00)$ & 2.20 & 0.332 & - \\
\hline & Alcohol drinking & $1.00(1.00-1.00)$ & 6.608 & 0.037 & $\mathrm{PPD}<\mathrm{CIP}^{* *}$ \\
\hline & Cooking & $1.00(1.00-2.00)$ & 232.84 & $<0.001$ & 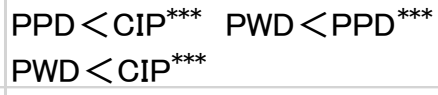 \\
\hline & Breakfast eating & $1.00(1.00-1.00)$ & 4.52 & 0.104 & - \\
\hline & Walking/exercise & $2.00(1.00-3.00)$ & 12.60 & 0.002 & $\mathrm{PPD}<\mathrm{CIP}^{* *}$ \\
\hline & Sleep duration/d & $2.00(2.00-2.00)$ & 64.10 & $<0.001$ & $\begin{array}{l}\mathrm{PPD}<\mathrm{CIP}^{* * *} \quad \mathrm{PWD}<\mathrm{PPD}^{*} \\
\mathrm{PWD}^{*}<\mathrm{CIP}^{* * *}\end{array}$ \\
\hline & Going out & $3.00(2.00-3.00)$ & 49.49 & $<0.001$ & $\mathrm{PPD}<\mathrm{CIP}^{* * *} \mathrm{PWD}<\mathrm{CIP}^{* * *}$ \\
\hline & Daytime lying duration & $1.00(1.00-1.00)$ & 99.27 & $<0.001$ & $\mathrm{PPD}<\mathrm{CIP}^{* * *} \quad \mathrm{PWD}<\mathrm{CIP}^{* * *}$ \\
\hline & Nr. of hobbies & $2.00(1.00-3.00)$ & 64.94 & $<0.001$ & $\mathrm{PPD}<\mathrm{CIP}^{* * *} \mathrm{PWD}<\mathrm{CIP}^{* * *}$ \\
\hline & Meat/poultry & $2.00(2.00-2.00)$ & 19.747 & $<0.001$ & $\mathrm{PPD}<\mathrm{CIP}^{* * *}$ \\
\hline & Soybean products & $2.00(1.00-2.00)$ & 28.343 & $<0.001$ & $\mathrm{PPD}<\mathrm{CIP}^{* * *} \mathrm{PWD}<\mathrm{CIP}^{* * *}$ \\
\hline & Eggs & $2.00(1.00-2.00)$ & 5.223 & 0.073 & - \\
\hline & Oily fish & $2.00(2.00-2.00)$ & 12.408 & 0.002 & $\mathrm{PPD}<\mathrm{CIP}^{* *} \mathrm{PWD}<\mathrm{CIP}^{*}$ \\
\hline & Dairy products & $1.00(1.00-2.00)$ & 8.753 & 0.013 & $\mathrm{PPD}<\mathrm{CIP}^{* *}$ \\
\hline & Fruits & $1.00(1.00-2.00)$ & 48.719 & $<0.001$ & $\mathrm{PPD}<\mathrm{CIP}^{* * *} \mathrm{PWD}<\mathrm{PPD}^{*}$ \\
\hline & Vegetables & $1.00(1.00-1.00)$ & 54.848 & $<0.001$ & $\mathrm{PPD}<\mathrm{CIP}^{* * *} \mathrm{PWD}<\mathrm{CIP}^{* * *}$ \\
\hline & Salted fish & $2.00(2.00-3.00)$ & 1.13 & 0.569 & - \\
\hline & Heavily seasoned food & $3.00(2.00-3.00)$ & 2.58 & 0.283 & - \\
\hline & Deep-/stir-fried food & $2.00(2.00-2.00)$ & 10.373 & 0.006 & $\mathrm{PPD}<\mathrm{CIP}^{* *}$ \\
\hline & Miso soup/soup & $3.00(2.00-3.00)$ & 6.10 & 0.047 & PWD $<\mathrm{CIP}^{*}$ \\
\hline & Pickled ume plums & $3.00(3.00-4.00)$ & 2.07 & 0.356 & - \\
\hline & Pickled vegetables & $2.00(1.00-3.00)$ & 36.20 & $<0.001$ & $\mathrm{PPD}<\mathrm{CIP}^{* * *} \mathrm{PWD}<\mathrm{CIP}^{* * *}$ \\
\hline
\end{tabular}

${ }^{\text {a. Kruskal-Wallis test, }}{ }^{\text {b. }} 3$ groups of PWD (people with dementia), PPD (people with probable dementia) and CIP (cognitively intact people).

Significance after Bonferroni correction: ${ }^{*} p<0.05 / 3=0.017,{ }^{* *} p<0.01 / 3=0.003,{ }^{* * *} p<0.001 / 3=0.0003$. 


\section{Discussion}

\subsection{BMI and the number of natural teeth}

PWD was the oldest and thinnest in men and women, respectively. This result suggests that the onset of dementia seemed to have appeared in the later years of the elderly and to have had under-nutrition and weight loss as Alzheimer's Disease International reported in 2014. The number of natural teeth might associate with this result. In men, the proportion of CIP who had $20-28$ natural teeth was $22.9 \%$, that of PPD $9.6 \%$ and that of PWD $12.5 \%$, while in women that of CIP was $21.7 \%$, that of PPD $5.0 \%$ and that of PWD $5.6 \%$. An association of dementia and the number of natural teeth was reported by following up 4,425 healthy elderly people over 65 years old from 2003 to 2007 (Yamamoto T, Kondo K, Hirai $\mathrm{H}$ et al., 2012). Among them the number of people with dementia under long-term care insurance was 220 (5.0\%). The elderly who had few teeth without dentures had a risk of the onset of dementia 1.9 times as much as those who had more than 20 natural teeth. In other words, the elderly who have more than 20 teeth are cognitively better like our CIP men and women. It is anticipated the number of natural teeth associates with better nutrition due to better mastication, and that our PWD men and women could have been the thinnest of the three groups.

Oral health is important to keep natural teeth to enjoy eating and promote quality of life and healthy longevity. WHO and FDI (Federation Dentaire Internationale) established the first Global Oral Health Goals jointly in 1981. In 1989 the then Ministry of Health and Welfare of Japan initiated "The 8020 Movement" which stands for "retaining 20 teeth at the age of 80 ". Life expectancy then was 75.91 in men and 81.77 in women, and so, at the beginning of the movement an attainment rate of "8020" was about $7 \%$ and the average number of remaining teeth was four to five. The attainment rates were improving like $15.3 \%$ in 1999 and $40.2 \%$ in 2010 . The latest Survey of Dental Diseases reports it was $51.2 \%$ in 2016. (Ministry of Health, Labour and Welfare, 2016). Further study will be required whether under-nutrition status will be improved in future PPD and PWD or not.

\subsection{Lifestyle}

\subsubsection{Alcohol drinking}

The group is scored from 1. rarely to 4. almost every day. The result suggests that some amount of alcohol is preferable. It was reported after reviewing 143 papers that the average ratio of cognitive risk (dementia or cognitive impairment and/or decline) associated with moderate "social" (not alcoholic) drinking of alcohol is 0.77 , with nondrinkers as the reference group, and that the benefit of moderate drinking applies to all forms of dementia (dementia unspecified, AD and Vascular Dementia) and to cognitive impairment (low test scores) (Neafsey EJ and Collons MA, 2011). In the questionnaires we asked the amount of alcohol drinking and to calculate the amount of alcohol in terms of Japanese rice wine 'sake'. 'Sake' is still measured using Japanese traditional measurement unit 'go' by custom. One go is $180 \mathrm{ml}$ in a metric unit and equivalent of $120 \mathrm{ml}$ 
of wine or $500 \mathrm{ml}$ of beer in pure alcohol (see Appendix 15-1). For convenience, converting into wine, among 649 male analysis subjects 196 men drank 120ml, 110 men 240ml, 22 men $360 \mathrm{ml}$ and 3 men $480 \mathrm{ml}$ of wine at a time, and among 787 female analysis subjects 62 women drank $120 \mathrm{ml}, 6$ women $240 \mathrm{ml}$ and 1 woman $360 \mathrm{mlof}$ wine at a time. One or two glasses of wine might be moderate social drinking as the previous study (Neafsey EJ and Collons MA, 2011) reported.

\subsubsection{Cooking}

The significant difference was observed between CIP and PPD in both men and women. Only in women it was observed between CIP and PWD. The elderly women have been taking or used to take cooking upon themselves as housewives for a long time, which suggested even PPD women could play their habitual role in their household.

Cooking a meal is a multi-functional act and requires several executive functions including planning, temporal sequencing and goal-attainment functions (Doherty TA, Barker LA, Denniss $\mathrm{R}$ et al., 2015). It is difficult for persons with dementia to cook meals and such results in women were clearly shown. Since cooking isn't a men's role in the days of our analysis subjects, it is understandable that significance was only shown between CIP and PPD in men.

\subsubsection{Walking/exercise}

As for walking/exercise, the significant differences were observed between CIP and PPD in men, and CIP and PPD and CIP and PWD in women. Our youngest group, the CIP men and women, could be easily assumed to go and enjoy walking/exercise. Walking/exercise bolsters up people's spirits, and it may bolster the brain function and skills of people with dementia. The previous study (Hsu CL, Best JR, Davis JC et al., 2017) looked into the effects of walking on vascular cognitive impairment (VCl) which is the second most common type of dementia after Alzheimer's disease. Ten older adults with mild subcortical ischemic $\mathrm{VCl}$ (SIVCI) were assigned to 'one-hour walking, 3 times a week for 6-month' at the university's lab. Participants were asked to move briskly enough during workouts to raise their heart rates to about 65 percent of their maximum capacity. The aerobic training group, compared with the control group $(n=11)$, significantly improved flanker task reaction time. They report their results suggest aerobic training among older adults with mild SIVCI can improve executive functions and neural efficacy of associated brain areas.

\subsubsection{Sleep duration per day including naps}

The results revealed the significant differences between CIP and PPD and CIP and PWD in men. In women they were shown between CIP and PPD, PPD and PWD and CIP and PWD. It is reported that sleep duration during the 24-hour was positively correlated with severity of dementia in nursing home patients. (Fetveit $A$ and Bjorvatn B, 2006). During the 24-hour period, patients spent nearly 13 hours asleep, distributed as more than ninehour of nighttime sleep and more than 3-hour of daytime sleep. Another study (Westwood 
AJ, Beiser A, Jain N, et al., 2017) reports that prolonged sleep duration was associated with an increased risk of incident dementia and sleeping for more than nine hours may be early sign of dementia. In this study the proportions of those who slept more than nine hours in men were $57.1 \%$ in PWD, 51.0\% in PPD and $21.3 \%$ in CIP and in women $73.3 \%$ in PWD, $44.0 \%$ in PPD and $15.1 \%$ in CIP.

\subsubsection{Going out, daytime lying duration in bed and the number of hobbies}

As for daytime lying duration in bed and the number of hobbies, the significant differences were observed between CIP and PPD and CIP and PWD in both men and women, however, as for going out they were shown between CIP and PPD in men, and between CIP and PPD and CIP and PWD in women. Items of daytime lying duration in bed, the number of hobbies and going out are associating each other. The common elements are social and mental activities and social network. People whose daytime lying duration in bed is prolonged do not enjoy hobbies and are difficult to go out. It is reported that social engagement and avoiding loneliness may have a protective effect on developing dementia (Pillai JA and Verghese J, 2009). A systematic review reports that actively participating in cognitive leisure activities during mid- or late life may be beneficial in preventing the risk of Alzheimer's disease and other dementias in the elderly (Stem C and Munn Z, 2010). In our analysis subjects, the largest proportions of those who are almost out of bed were 81.2\% in CIP, 43.8\% in PPD and 46.7\% in PWD in men, and in women they were $83.4 \%$ in CIP, 52.6\% in PPD and 31.3\% in PWD. As for going out, the tendency is observed that women went out less than men. The proportion of going out almost every day in women was $17.0 \%$ even in CIP, while it was $35.5 \%$ in CIP men. In women $23.4 \%$ of CIP went out 3-4 times per week and $44.7 \%$ of CIP went out once or twice per week. As for the number of hobbies in men, $51.6 \%$ had more than two and $19.7 \%$ had none in CIP, while no one had more than two and $75.0 \%$ had none in PWD. In CIP women $42.8 \%$ had more than two and $23.4 \%$ had none and in PWD women $15.4 \%$ had more than two and $80.8 \%$ had none.

\subsection{Dietary habits}

In women the significant differences were shown in nine dietary items except eggs, salted fish, heavily seasoned food and pickled ume plums. However, notable results were revealed in men. The significant difference was observed only in pickled vegetables out of 13 items. There are many kinds of pickled vegetables in Japan, among which the elderly prefer vegetables pickled in a fermented medium of rice bran and brine better than simply pickled in vinegar and salt. This result suggests that elderly women as housewives had been preparing meals considering foodstuffs and nutrition for their family members and they themselves ate balanced diet without likes and dislikes, while men ate meals own way.

Food items, in which significant differences were observed in women such as meat/poultry, eggs, dairy products, fruits and vegetables, are recommended by Alzheimer's Society (Food and health - Food for Thought) and widely recognized they are essential to keep 
good health condition. We would like to discuss focusing on fermented foods and oily fish which are traditionally consumed a great deal in Japan.

Fermented foods are reported to provide health benefits (Sanlier N, Gokcen BB and Sezgin AC, 2017) and consumption of the fermented soybean products is expanding. Frequently used ones are fermented soybean paste, which is 'miso' in Japan and 'doenjang' in Korea. A recent study of Hiroshima University suggests that Miso may have protective effects against stroke despite its high salt content (Watanabe $H$, Sasatani $M$, Doi $O$ et al., 2017). The doenjang supplementation was reported to have significant reductions in body weight and visceral fat in overweight adults (Cha YS, Yang JA, Back $\mathrm{HI}$, Kim MG et al. 2012). Due to their various health benefits and the popularity of Japanese foods since Japanese Cuisine (Washoku) was registered UNESCO's Intangible Cultural Heritage in 2013, exports of miso increased in late 2017 by $7.9 \%$ over the same period of the previous year (The Japan Agricultural News, Jan. 8, 2018). Discussing of fermented soybean paste, health benefits of isoflavones (daikzein and genistein) should be taken into consideration. The top four foods of soybean products are tofu (soybean curd), miso, natto (fermented soybeans) and fried tofu. They are popular foods and sources of isoflavones among Japanese. They covered about $90 \%$ of the population intake of daizein and genistein (Wakai K, Egami I, Kawamura T et al., 1999). Another study of the effects of various levels of natto intake, which contains plenty of vitamin $\mathrm{K} 2$, on bone stiffness and bone turnover markers in healthy premenopausal women reports that natto intake may have contributed to the promotion of bone formation (Katsuyama $\mathrm{H}$, Ideguchi S, Fukunaga $\mathrm{M}$ et al., 2004).

As for oily fish, previous studies clarify the health benefits of fatty fish on dementia. Laitinen et al. reports, after follow-up of 1,449 samples for 21 years on average, moderate intake of unsaturated fats at midlife is protective, whereas a moderate intake of saturated fats may increase the risk of dementia and AD (Laitinen MH, Ngandu T, Rovio S et al., 2006). Another study reports that consumption of fatty fish was associated with a reduced risk of dementia and $A D$ comparing associations of lean fish vs. fatty fish (tuna or other fish) intake with dementia, AD and VaD (Huang TL, Zandi PP, Tucker KL et al., 2005).

In this study, the significant differences were observed in soybean products, miso soup/soup and pickled vegetables in women. In soybean products and pickled vegetables, they were shown between CIP and PPD and CIP and PWD, and in miso soup/soup between CIP and PWD. The results suggest CIP women were healthier than PPD and PWD women.

Besides fermented food and oily fish, food items in which significant differences were observed in our subject women were meat/poultry, eggs, dairy products, fruits and vegetables, which are recommended by Alzheimer's Society as described above. These are the key ingredients of the Mediterranean diet (MeDi) which have been eaten in the area bordering the Mediterranean Sea including Greece, Italy, Spain, Morocco and others and was registered UNESCO's Intangible Cultural Heritage in 2010, three years earlier 
than Japanese "Washoku". Ingredients are similar to Japanese cuisine. Findings in our analysis women are overlapped with the benefits of MeDi of fruits, vegetables, beans, oily fish, dairy products, meat/poultry with moderate amount of alcohol drinking. According to systematic reviews, there are many reports that adherence to MeDi was associated with better cognitive function, lower rates of cognitive decline and reduced risk of AD (Solfrizzi V et al. 2011; Lourida I et al. 2013; Singh B et al. 2014). Mayo Clinic, Minnesota, USA, recommends Mediterranean diet and introduces its benefits for healthy eating to reduce the risk of heart disease, overall mortality and an incidence of cancer and AD (Mayo Clinic, 2018). MeDi includes yogurt which is also a fermented food. Thus, Japanese cuisine and Mediterranean diet have a lot in common in healthy dietary items.

\section{Concluding Remarks}

Since dementia is a priority issue worldwide, it is significant to clarify healthy lifestyle and dietary factors associating with dementia status. Japanese women enjoy the world longest longevity for 30 years, though including areas the latest record was established by Hong Kong women. It is worth to review Japanese elderly lifestyle and dietary habits in the association with their cognitive and dementia status to find a way of dementia risk reduction and to lead a quality life.

Briefly reviewing this study, the number of analysis subjects was 1,436 elderly men and women aged 65 year and older, 52 people with dementia (PWD), 173 people with probable dementia (PPD) and 1,211 cognitively intact people (CIP). Mean weight and BMI of analysis subjects by dementia status were shown that PWD was the oldest and thinnest in men and women, respectively. This suggests that the onset of dementia seemed to have appeared in the later years of the elderly and to have had under-nutrition and weight loss as Alzheimer's Disease International reported in 2014.

Descriptive statistics, a chi-square, Kruskal-Wallis and Mann-Whitney $U$ tests and Bonferroni's multiple comparisons were performed in men and women, respectively, significance set at 0.05 (0.017 after Bonferroni correction). Significant differences were shown in alcohol drinking, cooking, walking/exercise, sleep duration per day including naps, going out, daytime lying duration in bed and the number of hobbies in both men and women. As for dietary items in women they were observed in nine items which were meat/poultry, soybean products, oily fish, dairy products, fruits, vegetables, deep-/stir-fried food, miso soup/soup and pickled vegetables which are recommended by Alzheimer's Society, however, in men only pickled vegetables is clarified to have a significant difference. In discussion we paid a special attention on fermented soybean products which are frequently eaten and sources of isoflavones among Japanese. Oily fish is healthy and beneficial as fatty fish on dementia. Dietary items whose significant differences were observed are similar to ingredients of Mediterranean diet. Traditional foods eaten by the elderly in Japan and countries surrounded Mediterranean Sea have a lot in common.

The CIP women showed better health behavior in both lifestyle and dietary habits, while even the CIP men showed worse health behavior especially in dietary habits. In terms of 
health promotion and risk reduction of developing dementia, men should be more conscious of food intake. This study implies cognitive decline and dementia risk were associated with the number of natural teeth, foods (especially soybean products, oily fish and fermented foods), exercises, social interaction and moderate amount of alcohol drinking. It is also suggested that prolonged sleep duration more than nine hours per day might be an index of early detection of dementia status for family caregivers. However, the results of this study was limited in one population-based investigation of a small town. We need a comparative study between Town B and another city to investigate if similar factors to reduce risk of developing dementia can be found or not. It will be our next task.

\section{Appendix}

Questionnaire of the 2004 Complete Survey of the Community-dwelling Elderly of 65 and Older in Town B

We would like to ask the addressee to answer this questionnaire. When the addressee cannot answer by himself or herself, a proxy may fill in it. Please check the appropriate number. If you choose "Other", please fill in specific details in the parentheses.

\section{About an addressee}

1. Who answers this questionnaire?
1. The addressee
2. A proxy (family member)

2. What are the reasons when a proxy answers?

$\begin{array}{lll}\text { 1. Dementia (poor understanding) } & \text { 2. Hearing impairment } & \text { 3. Vision impairment }\end{array}$
4. Speech impediment
5. Sick/injured
6. Busyness
7. Absence

8. Doesn't want to answer. 9. Other ( )

3. Who do you live with now? Please check all appropriate answers.
1. Living alone
2. Spouse
3. Son/daughter
4. Son's wife
5. Grandchild
6. Other (
)

\section{About health}

4. What do you think of your health? Please check just one.
1. Very healthy
2. Reasonably healthy
3. Not so healthy
4. Not healthy

5. Do you have a health check-up once a year?

1. Yes 2. No

6. Do you feel pain in any part of your body? Please check all appropriate parts.
1. Neck
2. Shoulder(s)
3. $\operatorname{Arm}(\mathrm{s})$
4. Lower back
5. Knee(s)
6. Ankle(s) 

7. Other (
) 9. No pain

7. How many natural teeth do you have in all? Please fill in the number of them.

I have ( ) natural teeth.

8. Do you have diseases under treatment now? Please check all that apply.

1. High blood pressure 2. Stroke (cerebral infarction, cerebral hemorrhage, subarachnoid hemorrhage, etc.) 3. Hyperlipidemia 4. Diabetes mellitus

5. Heart disease (myocardial infarction, angina, arrhythmia, etc.) $\quad 6$. No diseases

9. Did (Do) your family members have diseases above? Please check all that apply.

1) Grandfather: $1 . \quad$ 2. 3. 4. 5. 6.

2) Grandmother: $1 . \quad$ 2. 3. 4. 5. 6.

3) Father: $1 . \quad 2 . \quad 3 . \quad 4 . \quad 5.6$.

4) Mother: 1. 2. 3. 4. 5. 6.

5) Brothers/sisters: 1. 2. 3. 4. 5. 6.

\section{Present diet, tobacco and alcohol}

10. Present diet

Using the following scale of 1 to 4 , please rate how often you eat the food inquired.
1. Almost every day
2. Sometimes
3. Rarely
4. Never

1) Do you eat meat/poultry?

$1-2-3-4$

2) Do you eat soy products like tofu and natto (fermented soybeans)? $1-2-3-4$

3) Do you eat eggs?

$1-2-3-4$

4) Do you eat oily fish like sardine, mackerel and Pacific saury?

$1-2-3-4$

5) Do you eat dairy products like cheese, yoghourt and milk?

$1-2-3-4$

6) Do you eat fruits?

$1-2-3-4$

7) Do you eat (cooked) vegetables?

$1-2-3-4$

8) Do you eat salted fish like salted salmon and cod and dried fish?

$1-2-3-4$

9) Do you eat heavily seasoned food?

$1-2-3-4$ 
10) Do you eat deep-/stir-fried food?

11) Do you eat breakfast?
$1-2-3-4$

$$
1-2-3-4
$$

11. How many bowls of miso soup/soup do you eat daily?
1. More than 3 bowls
2. 2 bowls
3. 1 bowl
4. No bowls

12. How many pieces of pickled ume plum daily?
1. More than 3 pieces
2. 2 pieces
3. 1 piece
4. None

13. How often do you eat pickled vegetables daily?
1. More than 3 times
2. Twice
3. Once
4. None

14. Do you smoke?
1. Never smoked
2. Quitted
3. Smoking

15. Do you drink alcohol such as sake, beer, whisky and wine?
1. Rarely
2. Once or twice a week
3. 3-5 times a week
4. Almost everyday

15-1. Please calculate the amount of alcohol in terms of the sake (Japanese rice wine) following the instruction below.

$180 \mathrm{ml}$ of sake $=500 \mathrm{ml}$ of beer $=180 \mathrm{ml}$ of shochu (Japanese distilled spirits $)=120 \mathrm{ml}$ of wine $=60 \mathrm{ml}$ of whisky or brandy

I drink ( ) $\mathrm{ml}$ in terms of the sake.

\section{About daily life}

16. Do you cook?
1. Often
2. Sometimes
3. Rarely

17. How often do you have walking and exercise?
1. Almost every day
2. 3-5 times a week
3. Once or twice a week
4. Rarely

18. How many hours of sleep do you get including a nap daily?
1. Less than 6 hours
2. 7-8 hours
3. More than 9 hours

19. How often do you go out?
1. Almost every day
2. 3-4 times a week
3. Once or twice a week
4. Less than once a month

20. Do you have persons around you with whom you feel at ease? 

1. Many
2. Some
3. Few
4. None

21. Do you have persons around you who can do errands for you?
1. Many
2. Some
3. Few
4. None

22. Do you take care of your pets (dogs/cats)?
1. Often
2. Occasionally
3. Rarely
4. Never

23. How long are you lying in bed during daytime?

1. I am almost out of bed. 2. I am out of bed longer than in bed.

3. I am in bed longer than out of bed. 4. I am almost in bed.

\section{About your hobbies and community activities}

24. How many hobbies do you have?
1. More than
2. One
3. None

25. Do you take part in community activities, volunteer work or activities with your friends?
1. Often
2. Occasionally
3. Rarely

26. Do you associate with your friends, colleagues and neighbors?
1. Almost every day
2. 3-4 times a week
3. Once or twice a week

4. Less than once a month

27. Do you enjoy trips and outing?
1. Often
2. Occasionally
3. Rarely

\section{About activities of daily living}

28. Please check 'yes' or 'no' about the following activities. (Please check 'yes' if you think you can do although you usually don't do it.)

1) Can you use public transportation (bus or train) by yourself?

1. Yes 2. No

2) Are you able to shop for daily necessities?

$\begin{array}{ll}\text { 1. Yes 2. No } & \text { 2 }\end{array}$

3) Are you able to prepare meals by yourself?

1. Yes 2. No

4) Are you able to pay bills?

1. Yes 2. No

5) Can you handle your own banking?

1. Yes 2. No

6) Are you able to fill out forms for your pension?

1. Yes 2. No 

7) Do you read newspapers?
1. Yes 2. No
8) Do you read books or magazines?

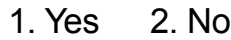
9) Are you interested in news stories or programs dealing with health?
$\begin{array}{ll}\text { 1. Yes } & \text { 2. No }\end{array}$
10) Do you visit the homes of friends?
$\begin{array}{ll}\text { 1. Yes } & \text { 2. No }\end{array}$
11) Are you sometimes called on for advice?
1. Yes 2. No
12) Are you able to visit sick friends?
1. Yes 2. No
13) Do you sometime initiate conversation with young people?
1. Yes 2. No

\section{About your occupation}

29. Please check the most appropriate answers about your job which you engaged longest.

- Occupation: 1. Office worker/public employee/association employee

2. Agricultural worker (including helper)

3. Forestry worker (including helper)

4. Self-employed worker (Type of business:

5. Part timer (Type of business:

6. Unemployed (retired)

7. Mainly housekeeping (including housewife)

8. Other (

-Working form: 1. Day duty

2. Night duty

3. Shift work

4. Other (

-Working hours: Approximately ( ) hours a day on average.

- The number of days off: ( $\quad$ ) days a month on average.

30. Did you enjoy (Are you enjoying) the occupation you checked?
1. I did (am).
2. I did (am) in my own way.
3. I hardly did (am).
4. Never

31. Could (Can) you adjust the amount and the deadline of work by yourself?
1. I could (can).
2. I could (can) in my own way.
3. I hardly could (can).

4. Never.

32. Are you satisfied with your life so far?

1. Yes. 2. No. 3. It is hard to say. 
33. What was your income last year including your spouse's (in Japanese yen)? Please include pension and financial support from your children if any.

1. No income

3. 1 million-less than 2 million yen

5. 3million-less than 4 million yen

7. 5million-less than 6 million yen

9. 7 million-less than 8 million yen

11. 9 million-less than 10 million yen

12. I don't want to answer.
2. Less than a million yen

4. 2 million-less than 3 million yen

6. 4 million-less than 5 million yen

8. 6 million-less than 7 million yen

10. 8 million-less than 9 million yen

11. More than 10 million yen

34. Attribute of the addressee.

- Age: ( ) year-old

- Weight: (

) $\mathrm{kg}$

- Maximal Blood Pressure: (

\section{- Sex: 1. Male 2. Female}

- Height: ( ) cm

- Weight in the 20s: Approximately ( ) kg.

We would appreciate it if you would check once more that you have not omitted anything.

Thank you very much for your cooperation. 


\section{References}

Asada T, Ikeda M, Kiyohara Y, et al. (2013): Research Report 2011-2012: Prevalence of dementia in urban areas and measures for daily living disorders caused by dementia (in Japanese). March 2013. www.tsukuba-psychiatry.com/up-content/uploads/2013/06/H24Report Part1.pdf (Accessed on June 24, 2017).

Alzheimer's Disease International (2015): World Alzheimer Report 2015, The Global Impact of Dementia, An ANALYSIS OF OREVALENCE, INCIDENCE, COST AND TRENDS.

Alzheimer's Society. Food and health - Food for Thought.

http://www.alzheimers.org.uk/site/scripts/documents info.php?documentID=1614

Cabinet Office (2017): The White Paper of Aged Society 2017, (in Japanese). http://www8.cao.go.jp/kourei/whitepaper/w-2017/zenbun/29pdf index.html (Accessed on January 22, 2018).

Cha YS, Yang JA, Back HI, Kim MG et al. (2012): Visceral fat and body weight are reduced in overweight adults by the supplementation of Doenjang, a fermented soybean paste. Nutr Res Pract. 6(6); 520-6. Doi: 10.4162/nrp.2012.6.6.520.

Dementia Supporters Caravan (2017). www.caravanmate.com (Accessed on June 24, 2017).

Doherty TA, Barker LA, Denniss R et al. (2015): The cooking task: making a meal of executive functions. Frontiers in Behavioral Neuroscience; 9:22. Doi: 10.3389/fnbeh.2015.00022.

Fetveit A, Bjorvatn B, 2006): Sleep duration during the 24-hour day is associated th the severity of dementia in nursing home patients. Int J Geriatr Psychiatry; 21(10):945-50. Doi: 10.1002/qps.1587.

G8 DEMENTIA SUMMIT DECLARATION (2013): https://www.gov.uk/government/publications/g8dementia-summit-agreements/g8-dementia-summit-declaration (Accessed on May 7, 2017).

G7 Ise-Shima Vision for Global Health (2016): www.mofa.go.jp/files/000160273.pdf (Accessed on May 7, 2017).

Hsu CL, Best JR, Davis JC et al. (2017): Aerobic exercise promotes executive functions and impacts functional neural activity among older adults with vascular cognitive impairment. British Journal of Sports Medicine; pil: bjsports-2016-096846. Doi: 10.1136/bjsports-2016-096846.

Huang TL, Zandi PP, Tucker KL et al. (2005): Benefits of fatty fish on dementia risk are stronger for those without APOE epsilon4. 65(9); 1409-14. Doi: 10.1212/01.wnl.0000183148.34197.2e.

Katsuyama H, Ideguchi S, Fukunaga M et al. (2004): Promotion of bone formation by fermented soybean (Natto) intake in premenopausal women. J Nutr Sci Vitaminol (Tokyo). 50(2); 114-20. https://doi.org/10.3177/jnsv.50.114

Koyano W, Shibata H, Nakazato K, et al. (1987). Tokyo Metropolitan Institute of Gerontology Index of Competence (TMIG-Index of competence): Measurement of competence in the elderly living at home 
(article in Japanese). Jpn J Public Health; 34:109-114.

Laitinen MH, Ngandu T, Rovio S et al. (2006): Fat intake at midlife and risk of dementia and Alzheimer's disease: a population-based study. Dement Geriatr Cogn Disord. 22(1); 99-107.

Doi: $10.1159 / 000093478$.

Ministry of Health, Labour and Welfare (2016): Dental Disease Survey 2016. http://mhlw.go.jp/toukei/list6228.html (Accessed on January 28, 2018).

Ministry of Health, Labour and Welfare (2017): Annual Health, Labour and Welfare Report 2016. www.mhlw.go.jo/english/wp/wp-hw10/dl/01e.pdf (Accessed on June 23, 2017).

Nakamura S, Shigeta M, Iwamoto M, et al. (2004): Prevalence and predominance of Alzheimer type dementia in rural Japan. Psychogeriatirics; 3(3): 97-103. https://doi.org/10.1111/j.1479-8301.2003.00020.x

Neafsey EJ, Collons MA (2011): Moderate alcohol consumption and cognitive risk. Neuropsychiatric Disease and Treatment; 7(1):465-484. https://doi.org/10.2147/NDT.S23159

Pillai JA, Verghese J. (2009): Social networks and their role in preventing dementia. Indian J Psychiatry; 51(Suppl1): S22-S28.

Sanlier N, Gokcen BB, Sezgin AC. (2017): Health benefits of fermented foods. Crit Rev Food Sci Nutr. 25:122. Doi: 10.1080/10408398.2017.1383366. (Accessed on Feb.10, 2018)

Stem C, Munn Z. (2010): Copgnitive leisure activities and their role in preventing dementia: asystematic review. Int J Evid Based Healthc. 8(1); 2-17. Doi: 10.1111/j/1744-1609.00150.x.

The Japan Agricultural News: Jan. 8, 2018.

Wakai K, Egami I, Kawamura T et al. (1999): Dietary intake and sources of isoflavones among Japanese. Nutr Cancer. 33(2); 139-45. Doi: 10.1207/S15327914NC330204.

Watanabe H, Sasatani M, Doi O et al. (2017): Protective Effects of Japanese Soybean Paste (Miso) on Stroke in Stroke-Prone Spontaneously Hypertensive Rats (SHRSP). American Journal of Hypertension, 31(1); 43-47. Doi: 10.1093/ajh/hpx1.29.

Westwood AJ, Beiser A, Jain N, et al. (2017): Prolonged sleep duration as a marker of early neurodegeneration predicting incident dementia. Neurology; 88(12):1172-1179.

Doi:10.1212NNL.0000000000003732.

Yamamoto C and Hoshi T (2010): Proportion of overlooked dementia in the community-dwelling elderly: the relationship between cognitive impairment and 5.9-year survival in an urban population (article in Japanese). Journal of Health and Welfare Statistics, 2010; 57(6): 18-24.

Yamamoto C and Hoshi T (2012): Steadily decreasing prevalence of dementia in Japanese urban elderly during a six-year follow-up. Proceedings of the $27^{\text {th }}$ International Conference of Alzheimer's Disease International, London. 21-124. 
Yamamoto T, Kondo K, Hirai H et al. (2012): Association between self-reported dental health status and onset of dementia: AGES project 4-year prospective cohort study of older Japanese. Psychosomatic Medicine 74(3):241-248. https://doi.org/10.1097/PSY.0b013e318246dffb 